\title{
Spectrum Sharing Technologies for Cognitive IoT Networks: Challenges and Future Directions
}

\author{
Bekele M. Zerihun ${ }^{1 *}$, Thomas O. Olwal' ${ }^{2}$ Murad R. Hassen ${ }^{1}$ \\ ${ }^{I}$ School of Electrical and Computer Engineering, AAiT, AAU, Addis Ababa, Ethiopia \\ ${ }^{2}$ Graduate School of Electrical and Computer Engineering, TUT, Pretoria, SA
}

Received: 16 December 2019; Accepted: 31 December 2019; Published: 08 February 2020

\begin{abstract}
In recent years, the number of devices connected to the Internet has been increased exponentially, which creates a new ecosystem known as the Internet of Things (IoT). According to Cisco's prediction, it is expected that over 50 billion devices will be connected to the Internet by 2020. In fact, IoT is expected to be a key enabling technology to accommodate the massive connectivity of heterogeneous smart devices in the upcoming fifth-generation networks. However, in a limited resource environment, the existing spectrum will not be sufficient to satisfy all these spectrum demand. Sharing the idle spectrum in licensed and unlicensed bands is a feasible solution for effective IoT deployment. Therefore, in this paper, we provide a survey of advanced spectrum sharing techniques and emerging IoT technologies to exploit the spectrum both in existing licensed cellular infrastructures and unlicensed spectrum bands. To the best of our knowledge, different from the existing literature, we identify the potential research challenges and suggest future directions for efficient IoT deployment in next-generation wireless networks.
\end{abstract}

Index Terms: Spectrum sharing technologies, Internet of Things, next-generation networks, cognitive radio

(C) 2020 Published by MECS Publisher. Selection and/or peer review under responsibility of the Research Association of Modern Education and Computer Science

\section{Introduction}

The recent advancements of ultra-low-power communication technologies, their continued fall in prices, and high computational capabilities are the driving factors for massive deployment of embedded systems, which creates the new Internet usage known as the Internet of Things (IoT) [1]. IoT is an intelligent infrastructure that targets transforming every physical object to be connected through the Internet. These physical objects are built

* Corresponding author

E-mail address: bekele.mulu@aait.edu.et 
with sensors and capable of interpreting their environment to communicate with each other without or with minimum human involvement [2].

In fact, the future wireless communication system is expected to meet the ambitious $5 \mathrm{G}$ requirements such as billions of devices connected to the Internet [3]. As shown in Fig. 1, Cisco predicts that over 50 billion devices will be connected to the Internet by 2020 [4]. A large share of these will be applications served by short-range radio technologies such as $\mathrm{Wi}-\mathrm{Fi}$ and Bluetooth, while a significant proportion will be enabled by low power wide area networks (LPWANs).

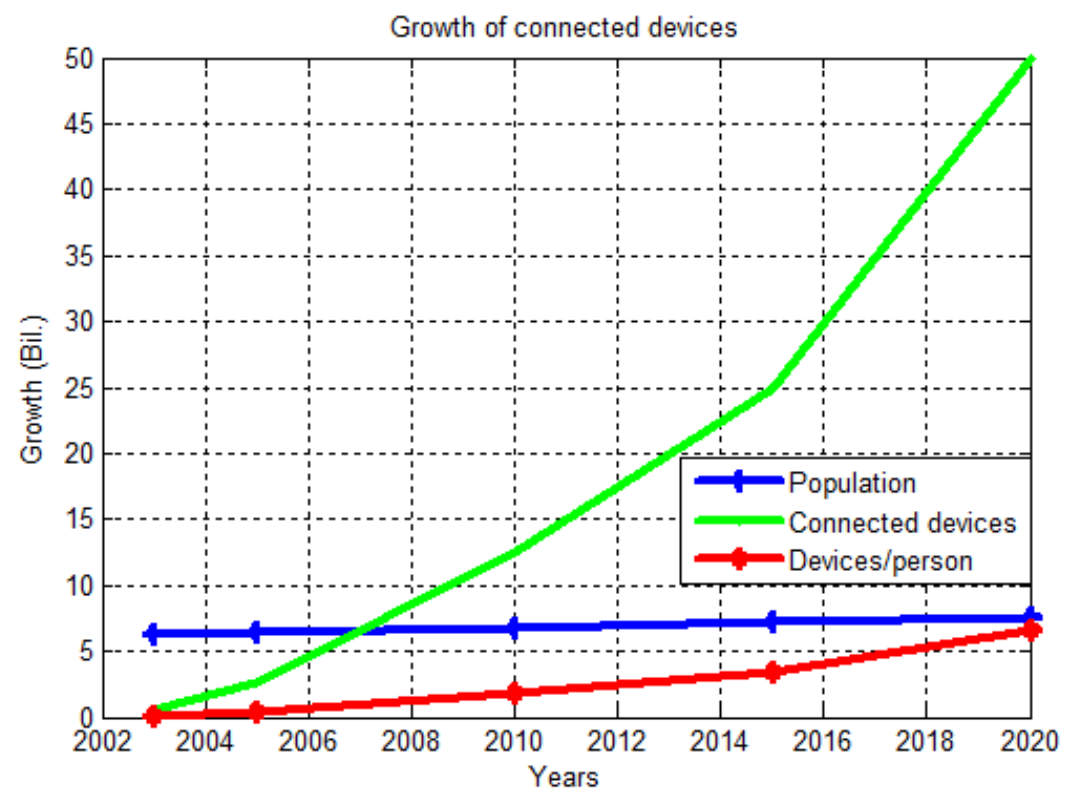

Fig. 1. Growth of devices connected to the Internet (4)

From Fig. 1, it is observed that the number of devices per person will be equivalent to the population and even surpass in the near future. In this networked society, spectrum scarcity could be a major challenge to satisfy the massive device connectivity. Therefore, the spectrum scarcity issue is required to be studied in prior to accommodate this massive device connectivity in next generation networks [5]. Spectrum sharing is considered as a key enabler to address the trade-offs between the scarce spectrum and deployment of a large number of IoT devices [6].

Indeed, the third generation partnership project (3GPP) has made significant enhancements to satisfy the demands of emerging IoT services and applications. The newly proposed solutions include extended coverage GSM (EC-GSM), narrowband IoT (NB-IoT), and long-term evolution for machine type communication (LTEM). In particular, NB-IoT and LTE-M are two basic IoT technologies that meet 3GPP Release 13/14 standards [7]. On the other hand, mobile operators are inclined to deploy IoT devices in the unlicensed spectrum with low power radio access technologies to reduce license related costs. The most common low power radio access technologies which operate in sub-GHz unlicensed bands are LoRaWAN and SigFox [8].

In this paper, different from the existing literature, we present a comprehensive survey of efficient spectrum sharing methods mainly focused on new radio network known as cognitive radio-enabled low power wide area network (CR-LPWAN). In particular, we identify major research challenges and suggest future directions related to spectrum sharing technologies for efficient IoT deployment in next generation wireless networks. 


\section{Related Works}

Recently, spectrum regulatory authorities have introduced new licensing models that pave the way toward dynamic spectrum sharing between multiple network operators [9]. With dynamic spectrum sharing scheme, users can access a common pool of spectrum resources with different priority as primary and secondary users [10]. There have been several studies on spectrum sharing techniques for unlicensed users to dynamically access the licensed bands in cognitive radio networks. In [11], the authors presented an approach to solve research challenges in the field of CR technology for IoT. Authors reviewed CR techniques to address various problems of IoT including energy efficiency, scalability and network heterogeneity. A service prioritized scheduling scheme for IoT is presented in [12]. The authors proposed traffic modeler to support proposed scheme. The proposed model is the service-centric spectrum usage pattern of IoT nodes as a six-state continuous-time Markov chain. Xing et al. [13] present the continuous-time Markov models of spectrum etiquette for dynamic spectrum access in open spectrum wireless networks. A random access protocol is proposed in order to show the achievement of airtime fairness. These channel access protocols are extended to spectrum agile radios. Sun et al. [14] investigated a novel CR application in IoT networks to address the concerns of spectrum scarcity. As IoT network interconnects several devices, it presents new challenges, such as available spectrum inadequacy, frequent device connection failures, and high energy consumption. Etkin et al. [15] proposed a non-cooperative game for the distributed dynamic spectrum sharing, where all users are selfish and do not reveal their private information. Under this model, the authors study the spectrum sharing problem among multiple secondary users for interference-constrained wireless systems in a non-cooperative game framework. Furthermore, authors in [16] designed a novel scheme for cooperative spectrum sensing and sharing based on the inspection game model for CR based IoT systems.

The existing literature have merely focused on conventional spectrum sharing technologies mainly developed for the downlink long packet communication from core network to end user devices. However, spectrum sharing in IoT networks will be mainly governed by uplink short packet communication from end user devices to core network. Therefore, the newly emerging spectrum sharing technologies for cognitive IoT applications and services must be revisited.

\section{Emerging Spectrum Sharing Technologies for IoT Applications and Services}

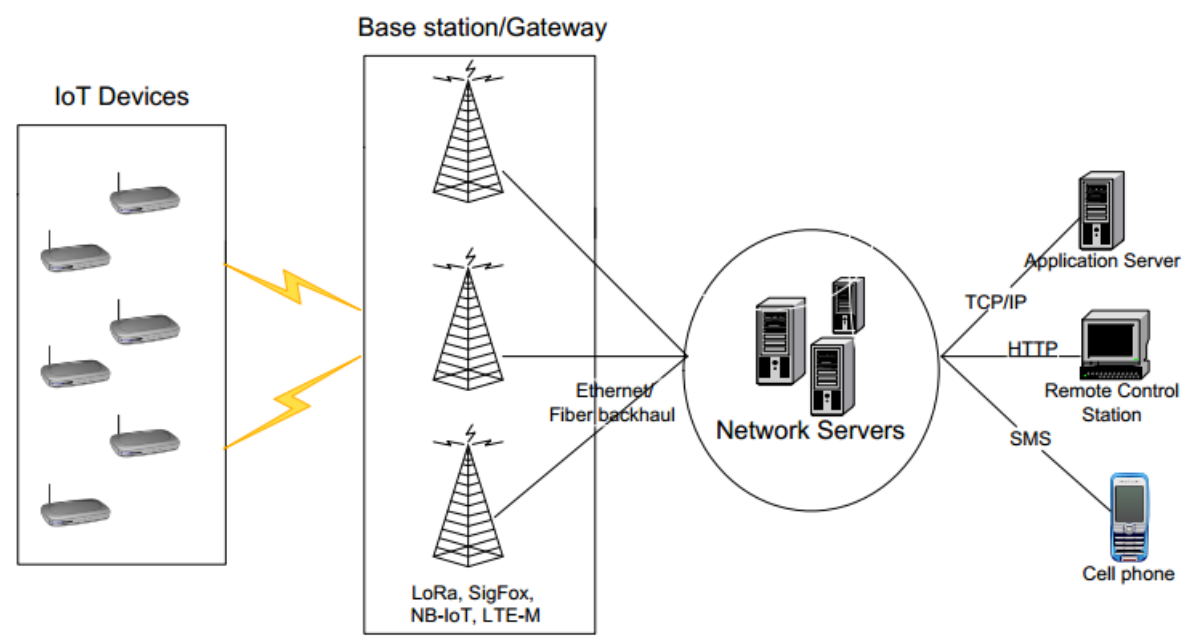

Fig. 2. A typical network architecture for cognitive IoT systems 
In recent years, a variety of networking and communication technologies are emerged to accommodate massive connectivity of heterogeneous devices. These technologies enable IoT devices to share the spectrum both in licensed and unlicensed bands [17]. However, emerging IoT applications and services have different architecture and protocol requirements to support two types of radio systems. Fig. 2 depicts the new architecture for cognitive IoT networks. In this architecture, IoT devices can communicate with the central entity using different communication technologies as a gateway.

Fig. 2 shows how the IoT devices can communicate with different applications and services through new spectrum sharing technologies as a gateway such as LoRa, SigFox, NB-IoT and LTE-M.

\subsection{NB-IoT and LTE-M}

- NB-IoT:

NB-IoT is representative IoT technology deployed within the licensed cellular spectrum for providing wide area coverage. Indeed, NB-IoT offers additional deployment flexibility by exploiting both legacy LTE spectrum and other re-farmed cellular spectra. Basically, NB-IoT is developed to be compatible with GSM and LTE technologies to achieve best performance in terms of coexistence [18]. A typical NB-IoT deployment scenario to share spectrum with GSM and LTE system is given in Fig. 3. NB-IoT needs a minimum of $180 \mathrm{kHz}$ bandwidth for both uplink and downlink, respectively. In fact, NB-IoT can be implemented in three different options; in its own designated spectrum (stand-alone), inside an LTE carrier (in-band), or in the guard band of LTE structure.

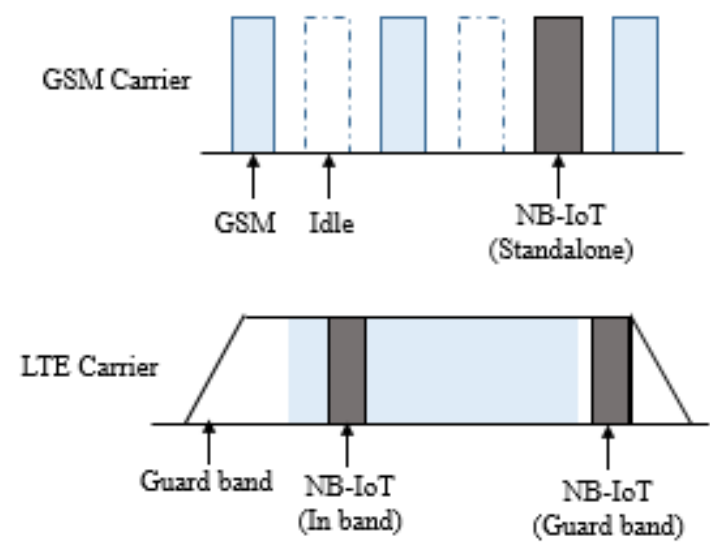

Fig. 3. Deployment scenario of NB-IoT to share the spectrum with GSM and LTE

- LTE-M:

LTE-M is a low-power wide area radio technology which supports IoT devices with low complexity and provides extended coverage, while allowing the reuse of the LTE infrastructure and share some common features. LTE-M protocol have similar spectrum structure and use same multiple access with legacy LTM [19]. However, LTE-M has a limitation in spectrum sharing since IoT devices can only exploit the in-band LTE spectrum in a centralized manner. Thus, further research investigation is required to dynamically access the idle spectrum in LTE network.

In general, both NB-IoT and LTE-M are promising technologies for the realization of massive connectivity of heterogeneous IoT devices in licensed cellular spectrum. A comparison of these two technologies with different parameters is clearly presented in Table 1. As seen from Table 1, both NB-IoT and LTE-M are good 
connectivity options for industries looking to take advantage of LPWAN technology. However, there are key differences between these two technologies, such as latency, bandwidth, data rate and maximum coupling loss (MCL).

Table 1. Comparative analysis of NB-IoT and LTE-M

\begin{tabular}{lll}
\hline Specification & NB-IoT & LTE-M \\
\hline Spectrum & $700-900 \mathrm{MHz}$ & $700-900 \mathrm{MHz}$ \\
Bandwidth & $1.08 \mathrm{MHz}$ & $180 \mathrm{kHz}$ \\
Data rate & $10 \mathrm{MHz}$ & $150 \mathrm{kHz}$ \\
Tx power & $23 \mathrm{dBm}$ & $23 / 35 \mathrm{dBm}$ \\
Latency & Low & Relatively high \\
MCL & $160 \mathrm{dBm}$ & $164 \mathrm{dBm}$ \\
\hline
\end{tabular}

\subsection{LoRaWAN and SigFox}

In addition to Bluetooth and Zigbee, new LPWAN technologies are deployed in unlicensed bands emerged into the market to facilitate the rollout of cognitive IoT networks. These networking and communication technologies are very important to enable connectivity among IoT devices and share the spectrum in unlicensed bands [8]. In the following, we discuss the main features, advantages and disadvantages of these technologies regarding with spectrum sharing solutions for IoT networks.

- LoRaWAN

LoRaWAN is a low-power, long range wireless technology designed for wide area applications. It allows low-power devices to communicate with each other through Internet for long range applications. LoRaWAN is an ideal technology for massive IoT devices to share the spectrum in unlicensed bands [20].

LoRaWAN standards define three device classes to reduce the energy consumption of IoT devices [21]. The three device classes are Class A, Class B and Class C. In Class A devices, a two-way communication between a gateway and a device is supported. The device transmits the uplink information randomly. Then it becomes ready to receive the feedback from any server by opening two receiving windows and wait for some specified time. If there is no response from any server in the given specified time, the receiving windows will be closed and wait an opportunity after the next uplink transmission. Class B devices have additional feature called scheduled receiving windows to receive information from the gateway/server. These devices open receiving windows periodically by using time-synchronized beacons transmitted from the server. In Class C devices, the receiving windows are left open to listen any response from the server unless they are transmitting the information. This feature allows to reduce the communication latency, while increase the energy consumption of the device.

To meet the spectrum regulation and avoid the interference with other wireless devices operating on the same channel, LoRaWAN is allowed to operate with a duty cycle not greater than $1 \%$. In addition, LoRaWAN uses chirp spread spectrum (CSS) technique and transmit a narrow band signal over a wideband channel [22]. LoRaWAN is a key technology to implement a large number of IoT devices in the unlicensed bands. However, further investigation is required to clarify the scope and major limitations of this technology in terms of channel hoping methods, multiple access techniques, feedback mechanisms, coordination systems between gateways from the same or different operators, and so on.

- $\quad$ SigFox

SigFox is another key technology for IoT devices to use very low bandwidth connections. SigFox is introduced in 2009 by a French global network operator. Compared to other technologies, it is relatively low- 
cost technology because it uses inexpensive radio modules. SigFox also supports a wide coverage area and it is a good candidate to accommodate a large number of IoT deployments over long ranges [23]. SigFox uses differential binary phase-shift keying (DBPSK) and the Gaussian frequency shift keying (GFSK) digital modulation techniques that enables it to communicate in the sub-GHz ISM bands. It utilizes a wide-reaching ultra-narrowband signal that can freely propagates through solid objects [8,23].

In general, SigFox is an ideal technology to accommodate a large number of IoT devices, because it consumes low power and has a wide network coverage. However, mobility is very difficult with SigFox technology. Therefore, further research investigation is required to properly utilize SigFox for efficient IoT spectrum sharing.

A general comparison of these two technologies with different parameters is depicted in Table 2 [23].

Table 2. Comparison of LoRaWAN and SigFox

\begin{tabular}{lll}
\hline Specification & LoRaWAN & SigFox \\
\hline MCL & $157 \mathrm{~dB}$ & $162 \mathrm{~dB}$ \\
Spectrum & $800-900 \mathrm{MHz}$ & $800-900 \mathrm{MHz}$ \\
Bandwidth & $125 \mathrm{kHz}$ & $0.6 \mathrm{kHz}$ \\
Data rate & $10 \mathrm{kHz}$ & $100 \mathrm{kHz}$ \\
Tx Power & $14 \mathrm{dBm}$ & $14 / 27 \mathrm{dBm}$ \\
\hline
\end{tabular}

\section{Research Challenges and Future Directions in IoT Spectrum Sharing}

The next generation radio access network, called 5G RAN is expected to be an integration of multiple evolved and revolved radio access technologies. This implies several research issues such as security, interference, network heterogeneity, network complexity needs to be further investigated to realize the spectrum sharing solutions in future IoT networks [24]. In this section, we identify potential research challenges and suggest future directions on how to address them.

\subsection{New Network Architecture}

The future IoT network architecture is required to deploy edge computing nodes close to IoT devices in order to meet latency requirements of these IoT devices. Data processing mechanism will be performed depending on the status of traffic density. Some data will be directly processed and respond to IoT devices by edge computing nodes. On the other hand, data from the farthest IoT devices will be semi-processed and filtered out at the edge computing nodes before transmitted to the base station [25]. However, re-designing the network architecture to support edge computing nodes made the IoT network very complex. Therefore, an alternative research direction in re-designing the future IoT network architecture is required to address the challenges related to their spectrum sharing solutions.

\subsection{Network Heterogeneity}

Network heterogeneity and traffic imbalance imposes service demand irregularity in future cellular networks. To reduce the power consumption of IoT devices and improve the network performance, a new traffic model is required. Indeed, spectrum sharing in heterogeneous network is quite different from conventional networks. In conventional homogeneous networks, spectrum sharing is designed for long-packet data transmission from base station to user equipment [26]. However, spectrum sharing in IoT networks may be designed for shortpacket uplink data transmission from terminal devices to base station. Therefore, it is inappropriate to directly apply conventional spectrum sharing techniques for IoT use cases. Thus, one potential research approach is to explore design and analysis of spectrum sharing for short-packet data transmission in the uplink. 


\subsection{Interference Management}

Interference is another major challenge in IoT spectrum sharing techniques. A critical threat to the reliability and efficient spectrum sharing in IoT is the interference generated by coexisted wireless networks [27]. Since most of IoT technologies are operate both in unlicensed and licensed bands, interference between these technologies will be severe. Therefore, a new design and implementation of interference mitigation technique is required in order to cancel the interference generated from co-located devices. Thus, it is necessary to explore new self-interference cancellation techniques for future IoT usages.

\subsection{Energy Efficiency}

In future wireless networks, operators may use millimeter-wave bands to satisfy its ultra-high traffic demands. Therefore, IoT devices may require to scan a wide range of spectrum to identify the overall channel status. However, this procedure will increase the energy consumption of IoT devices and affect the battery life time of user devices, especially for devices that have limited energy budgets [28]. Therefore, it is required to investigate an energy saving spectrum sensing mechanism in future IoT systems.

\subsection{Security}

In multi-carrier techniques such as NOMA system, different users may access multiple channels. In this case security issues will be raised as a critical problem. The cryptography based conventional security solutions are not satisfactory for future decentralized and heterogeneous networks [29]. Therefore, the design of physical layer security techniques is more suitable than cryptography-based approaches to realize advanced spectrum sharing in future IoT networks. Alternatively, imperfect self-interference cancellation shall be considered as a good research direction to investigate strong security techniques for efficient IoT spectrum sharing solutions.

\section{Conclusion}

With the exponential growth rate of smart devices connected to the Internet, spectrum scarcity will be a major challenge. In this paper, we present a comprehensive survey of advanced spectrum sharing techniques for massive heterogeneous IoT deployments in two types of radio networks. In particular, we overview emerging IoT technologies deployed both in licensed and unlicensed bands for IoT spectrum sharing. For each technology, we elaborate the basic features, specifications and their spectrum sharing solutions to enhance both energy and spectral efficiency. Finally, as a main contribution of this survey paper, we identify the major research challenges and suggest future directions for effective deployment of massive IoT devices in the coming $5 \mathrm{G}$ networks.

\section{References}

[1] Park D., Youn J. M., and Cho J., "A low-power microcontroller with accuracy-controlled event-driven signal processing unit for rare-event activity-sensing IoT devices," Journal of Sensors, vol. 2015, 2015.

[2] Sheng Z. et al., "A Survey on the IETF Protocol Suite for the Internet of Things: Standards, Challenges, and Opportunities," IEEE Wireless Commun., vol. 20, no. 6, Dec. 2013, pp. 91-98.

[3] Zerihun B. M. and Wondie Y., "Massive MIMO for 5G Cellular Networks: Potential Benefits and Challenges," In International Conference on Information and Communication Technology for Development for Africa, Springer, Cham, Sept. 2017, pp. 219-227. 
[4] Evans D., "The Internet of Things: How the Next Evolution of the Internet is Changing Everything," Cisco IBSG, Cisco White Paper, April 2011.

[5] Yang C. et al., "Advanced Spectrum Sharing in 5G Cognitive Heterogeneous Networks," IEEE Wireless Commun., vol. 23, no. 2, Apr. 2016, pp. 94-101.

[6] Ahmad A. et al., "A Survey on Radio Resource Allocation in Cognitive Radio Sensor Networks," IEEE Commun. Surveys and Tutorials, vol. 17, no. 2, 2015, pp. 888-917.

[7] "LTE evolution for IoT connectivity," Nokia, Tech. Rep. Nokia White Paper, January 2016. [Online]. Available: http://resources.alcatel-lucent.com/asset/200178.

[8] Nolan K. E. et al., "An Evaluation of Low Power Wide Area Network Technologies for the Internet of Things," In Proceedings of the International Wireless Communications and Mobile Computing Conference (IWCMC), Paphos, Cyprus, 5-9 September 2016.

[9] Irnich T. et al., "Spectrum Sharing Scenarios and Resulting Technical Requirements for 5G Systems," Proc. IEEE PIMRC Wksps., 2013, pp. 127-32

[10] RSPG, "Report on Collective Use of Spectrum (CUS) and Other Spectrum Sharing Approaches," RSPG 11-392, Nov. 2011.

[11] Rawat P., Singh K. D., and Bonnin J. M., "Cognitive radio for M2M and Internet of Things: A survey," Computer Communications, vol. 94, pp. 1-29, Nov. 2016.

[12] Eswaran S. P., and Bapat J., "Service centric markov based spectrum sharing for internet of things (IoT)," in IEEE Region 10 Symposium (TENSYMP), pp. 9-12, IEEE, May 2015.

[13] Xing Y., Chandramouli R., Mangold S., Shankar S., "Dynamic Spectrum Access in Open Spectrum Wireless Networks,” IEEE J. Sel. Areas Commun. 2006, 24, 626-637.

[14] Sunet D. al., "Spectrum sensing and the utilization of spectrum opportunity tradeoff in cognitive radio network," IEEE Commun. Lett., vol. 20, no. 12, pp. 2442-2445, Dec. 2016.

[15] Etkin R., Parekh A., Yse D., "Spectrum Sharing for Unlicensed Bands," IEEE J. Sel. Areas Commun. 2007, 25, 517-528.

[16] Kim S., "Inspection game based cooperative spectrum sensing and sharing scheme for cognitive radio IoT system," Computer Communications, vol. 105, pp. 116-123, Jun. 2017.

[17] M. Lauridsen et al., "Coverage and capacity analysis of LTE-M and NB-IoT in a rural area," In 2016 IEEE $84^{\text {th }}$ Vehicular Technology Conference (VTC-Fall), Sep. 2016, pp. 1-5.

[18] Ratasuk R. et al., "Narrowband LTE-M system for M2M communication," In 2014 IEEE $80^{\text {th }}$ Vehicular Technology Conference VTC2014-Fall), Sep. 2014, pp. 1-5.

[19] Hoglund A. et al., "Overview of 3GPP Release 14 enhanced NB-IoT," IEEE Network, vol. 21, no. 6, Nov. 2017, pp. 16-22.

[20] Naoui S. et al., "Enhancing the security of the IoT LoraWAN architecture," In 2016 International Conference on Performance Evaluation Modeling in in Wired and Wireless Networks (PEMWN), Nov. 2016, pp. 1-7.

[21] Sinha R.S., Wei Y., and Hwang S.H., “A Survey on LPWA technology: LoRa and NB-IoT,” Ict Express, vol. 3 no. 1, Mar. 2017, pp. 14-21.

[22] Reynders B. and Pollin S., "Chirp spread spectrum as a modulation technique for long range communication," In 2016 Symposium on Communications and Vehicular Technologies (SCVT), IEEE, Nov. 2016, pp. 1-5.

[23] Lauridsen M. et al., "Coverage Comparison of GPRS, NB-IoT, LoRa, and SigFox," In Proceedings of the IEEE Vehicular Technology Conference, Sydney, Australia, 4-7 June 2017.

[24] Olwal T. O., Djouani K., and Kurien A. M., "A Survey of Resource Management toward 5G Radio Access Networks," IEEE Communications, Surveys and Tutorials, vol. 18, no. 3, Apr. 2016, pp. 16561686.

[25] Uviase O., and Kotonya G., "Iot architectural framework: connection and integration framework for iot systems," 1st workshop on Architectures, Languages and Paradigms for IoT, 2018, pp. 1-17. 
[26] Zhang X., Cheng W., and Zhang H., "Heterogeneous statistical QoS provisioning over 5G mobile wireless networks," IEEE Networks, vol. 28, no. 6, 2014, pp. 46-53.

[27] Sparber T. et al., "Mitigating radio interference in large iot networks through dynamic cca adjustment," Open Journal of Internet of Things (OJIOT), vol. 3, no. 1, 2017, pp. 103-113.

[28] $\mathrm{Hu}$, Rose Q., and Qian Y., "An energy efficient and spectrum efficient wireless heterogeneous network framework for 5G systems," IEEE Communications Magazine, vol. 52, no. 5, 2014, pp. 94-101.

[29] Furqan H.M., Hamamreh J., and Arslan H., "Physical Layer Security for NOMA: Requirements, Merits, Challenges, and Recommendations," May 2019, arXiv preprint arXiv:1905.05064.

\section{Authors' Profiles}

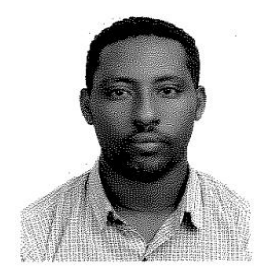

Mr. Bekele Mulu Zerihun received his B.Sc. and M.Sc. in Electrical Engineering in 2006 and 2014 respectively from Adama Science and Technology University and Bahir Dar University, Ethiopia. He is working as Lecturer in the department of Electrical and Computer Engineering, Wolaita Sodo University, Ethiopia and previously worked as a supervisor in Telecom projects. He is currently pursuing Ph.D. at Addis Ababa Institute of Technology (AAiT), Addis Ababa, Ethiopia. His research interests include spectrum sharing in cognitive radio networks, wireless sensor networks, Internet of Things, and software defined networks.

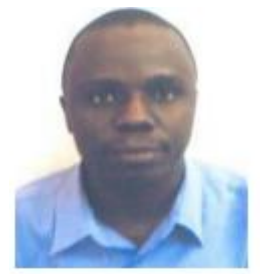

Prof. Thomas Otieno Olwal received his Ph.D. degree in computer science from the University of Paris-EST, Champs-sur-Marne, France, in 2010, and the D.Tech. degree in electrical engineering from Tshwane University of Technology (TUT) (in a cotutelle programme), Pretoria, South Africa, in 2011. He is currently lecturing at the TUT as a Professor and previously worked as a Senior Researcher with Wireless Computing and Networking R\&D, Council for Scientific and Industrial Research (CSIR). His research interests include analysis and design of the spectrum, energy-efficient radio resource management, Internet of Things, autonomous robotics, and automation and their emerging applications. He serves as a reviewer in a number of ACM/IEEE conferences and journals.

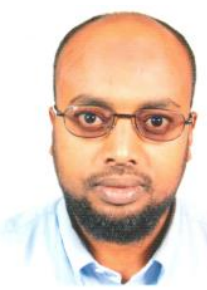

Dr. Murad Ridwan Hassen is a member, IEEE. He received his B.Sc, M.Sc and $\mathrm{PhD}$ in Electrical Engineering in 1993, 2001 and 2013, respectively from Addis Ababa Institute of Technology (AAiT), Addis Ababa University, Ethiopia. Currently he is working as Assistant Professor in the School of Electrical \& Computer Engineering, AAiT. He has publications in International and national Journals. His research interests include development of highly efficient algorithms for smart antenna design and analysis, and novel hybrid spectrum sharing for cognitive radio networks.

How to cite this paper: Bekele M. Zerihun, Thomas O. Olwal, Murad R. Hassen, " Spectrum Sharing Technologies for Cognitive IoT Networks: Challenges and Future Directions ", International Journal of Wireless and Microwave Technologies(IJWMT), Vol.10, No.1, pp. 17-25, 2020.DOI: 10.5815/ijwmt.2020.01.02 